\title{
First description of $A B C B 4$ gene deletions in familial low phospholipid-associated cholelithiasis and oral contraceptives-induced cholestasis
}

\author{
Eric Pasmant ${ }^{\star}, 1,2$, Philippe Goussard ${ }^{1}$, Laetitia Baranes ${ }^{1}$, Ingrid Laurendeau ${ }^{2}$, Samuel Quentin ${ }^{3}$, \\ Philippe Ponsot ${ }^{4}$, Yann Consigny ${ }^{5}$, Olivier Farges ${ }^{6}$, Bertrand Condat ${ }^{7}$, Dominique Vidaud ${ }^{1,2}$, Michel Vidaud ${ }^{1,2}$, \\ Jian-Min $\mathrm{Chen}^{8}$ and Béatrice Parfait ${ }^{1,2}$
}

The wide clinical spectrum of the $A B C B 4$ gene (ATP-binding cassette subfamily $B$ member 4) deficiency syndromes in humans includes low phospholipid-associated cholelithiasis (LPAC), intrahepatic cholestasis of pregnancy (ICP), oral contraceptivesinduced cholestasis (CIC), and progressive familial intrahepatic cholestasis type 3 (PFIC3). No ABCB4 mutations are found in a significant proportion of patients with these syndromes. In the present study, 102 unrelated adult patients with LPAC (43 patients) or CIC/ICP (59 patients) were screened for $A B C B 4$ mutations using DNA sequencing. Heterozygous $A B C B 4$ point or short insertion/deletion mutations were found in 37\% (16/43) of the LPAC patients and in $27 \%(16 / 59)$ of the ICP/CIC patients. High-resolution gene dosage methodologies were used in the 70 negative patients. Here, we describe for the first time $A B C B 4$ partial or complete heterozygous deletions in $7 \%(3 / 43)$ of the LPAC patients, and in $2 \%(1 / 59)$ of the ICP/CIC patients. Our observations urge to systematically test patients with LPAC, ICP/CIC, and also children with PFIC 3 for the presence of $A B C B 4$ deletions using molecular tools allowing detection of gross rearrangements. In clinical practice, a comprehensive $A B C B 4$ alteration-screening algorithm will permit the use of $A B C B 4$ genotyping to confirm the diagnosis of LPAC or ICP/CIC, and allow familial testing. An early diagnosis of these biliary diseases may be beneficial because of the preventive effect of ursodeoxycholic acid on biliary complications. Further comparative studies of patients with well-characterized genotypes (including deletions) and phenotypes will help determine whether $A B C B 4$ mutation types influence clinical outcomes. European Journal of Human Genetics (2012) 20, 277-282; doi:10.1038/ejhg.2011.186; published online 12 October 2011

Keywords: ABCB4; deletion; biliary disease; LPAC; MDR3

\section{INTRODUCTION}

ABCB4 (ATP-binding cassette subfamily B member 4) membrane transporter translocates phosphatidylcholine from the inner to the outer leaflet of the canalicular membrane of the hepatocyte. This floppase activity makes phosphatidylcholine available for extraction into the canalicular lumen by bile salts. The ABCB4 gene (MIM 171060) has a crucial role as evidenced by cholestatic liver diseases caused by its deficiency. ${ }^{1} A B C B 4$ is also known as multidrug resistance 3 gene (MDR3), a member of the MDR/TAP subfamily involved in multidrug resistance as well as antigen presentation. The human $A B C B 4$ gene is located on chromosome 7q21.1, contains 27 coding exons, and spans approximately $74 \mathrm{~kb}^{2}{ }^{2}$ The pathophysiology of the $A B C B 4$ alterations resides in the lack of phospholipid protection in the bile against the detergent effect of the bile salts, resulting in damage to the biliary epithelium, bile ductular proliferation, and potential progressive portal fibrosis. As biliary cholesterol solubilization depends not only on the concentration of the sterol itself but also on the bile salt and phospholipid concentration, a decreased rate of phospholipid excretion can also be a cause of gallstone formation.

The wide clinical spectrum of the $A B C B 4$ deficiency syndromes in humans covers cholestatic disorders presenting from the neonatal period of life to late adulthood. ${ }^{3-5}$ At least three distinct syndromes with variable severity have been clearly identified: progressive familial intrahepatic cholestasis type 3 (PFIC3; MIM 602347), low phospholipid-associated cholelithiasis (LPAC alias gallbladder disease 1, GBD1; MIM 60080), and familial intrahepatic cholestasis of pregnancy (ICP; MIM 147480). Evidences of $A B C B 4$ mutations have also been found in transient neonatal cholestasis, ${ }^{6}$ or adult idiopathic biliary fibrosis or cirrhosis. ${ }^{4,711}$

A recessive inheritance pattern of PFIC3 has been observed. ${ }^{7,12}$ Most $A B C B 4$ mutations in the patients with PFIC3 have been reported to be homozygous or compound heterozygous. ${ }^{3,7,9,12-14}$ These mutations include missense and non-sense mutations, and short frameshift deletions or insertions. $A B C B 4$ mutations are associated with an absence or a weak level of the canalicular ABCB4 protein, and with a low level of biliary phospholipids. ${ }^{72,15}$ Patients with PFIC3 usually

\footnotetext{
${ }^{1}$ Service de Biochimie et de Génétique Moléculaire, Hôpital Beaujon, Clichy, France; ${ }^{2}$ UMR745 INSERM, Université Paris Descartes, Sorbonne Paris Cité, Faculté des Sciences Pharmaceutiques et Biologiques, Paris, France; 'PPlateforme Génomique, Institut Universitaire d'Hématologie, Université Paris Diderot and Laboratoire d'Hématologie APHP, Hôpital Saint-Louis, Paris, France; ${ }^{4}$ Service de Gastroentérologie, Hôpital Beaujon, Clichy, France; ${ }^{5}$ Cabinet Médical, Paris, France; ${ }^{6}$ Service de Chirurgie digestive, Hôpital Beaujon, Clichy, France; ${ }^{7}$ Service d'Hépato-Gastroentérologie, Hôpital Saint-Camille, Bry-sur-Marne, France; 8 INSERM U613 and EFS-Bretagne, Brest, France

${ }^{*}$ Correspondence: Dr E Pasmant, UMR745 INSERM, Université Paris Descartes, Sorbonne Paris Cité, Faculté des Sciences Pharmaceutiques et Biologiques, 4 avenue de I'Observatoire 75006, Paris, France. Tel: +33 1537397 25; Fax: +33 1440717 54; E-mail: eric.pasmant@gmail.com

Received 29 June 2011; revised 8 September 2011; accepted 8 September 2011; published online 12 October 2011
} 
present at a few years of age and suffer from severe chronic and progressive cholestasis. Liver histology often reveals fibrosis with portal inflammation and strong bile duct proliferation in an early stage. ${ }^{16}$ A characteristic high-serum gamma-glutamyltransferase activity is found in PFIC3. As a consequence of the cirrhosis, the PFIC3 patients are prone to gastrointestinal bleeding. About $50 \%$ of the patients need a liver transplantation. Interestingly, the other half may benefit from treatment with ursodeoxycholic acid (UDCA). ${ }^{7}$

Mutations in the $A B C B 4$ gene that may reduce but not eliminate or drastically decrease the protein (leaving residual activity of the transporter), have been shown to cause a variety of milder cholestatic phenotypes, including LPAC and ICP. LPAC is a peculiar form of biliary gallstone disease characterized by intrahepatic sludge and/or symptomatic cholesterol cholelithiasis in young adults (usually before 40 years). LPAC is typically associated with mild chronic cholestasis, recurrence of symptoms after cholecystectomy, and prevention of recurrence by UDCA. About one third to half of patients have missense, frameshift, or non-sense mutations - mostly heterozygous - in the $A B C B 4$ gene. ${ }^{17-19}$ One of the hallmarks of LPAC is the response and remission induced by the UDCA therapy. Heterozygous $A B C B 4$ mutations were also identified in up to $15 \%$ of women suffering from ICP. ${ }^{12,20,21-31}$ ICP is a reversible form of cholestasis that may develop in the third trimester of pregnancy, usually rapidly resolves after delivery and recurs in $45-70 \%$ of subsequent pregnancies. $^{27}$ The main symptoms are pruritus and, to a lesser extent, jaundice. Serum bile salt and aminotransferases levels are elevated. Increased incidence of fetal complications (including placental insufficiency, premature labor, and sudden fetal death) was reported in association with ICP. UDCA is the treatment of choice for ICP and produces relief from pruritus with improvement in liver tests, with no adverse maternal or fetal effects. ${ }^{27,32,33}$ It is generally accepted that women who have suffered from ICP are also susceptible to the development of cholestasis on the use of oral contraceptives (oral contraceptives-induced cholestasis (CIC)). ${ }^{34,35}$ Evidences of $A B C B 4$ heterozygous mutations have also been found in patients with druginduced cholestasis or liver injury. ${ }^{17,18,35,36}$

Loss of function $A B C B 4$ mutations can present a large spectrum of cholestasis phenotypes, and genetic analysis of $A B C B 4$ can now be performed to confirm the diagnosis. ${ }^{37}$ However, no $A B C B 4$ mutations are found in a significant proportion of patients. The lack of $A B C B 4$ mutation detection in remaining cases might be attributed to phenotyping errors, genetic heterogeneity, and inadequacy of genetic screening methods. In the present study, we have tested the last hypothesis by using recent high-resolution gene dosage methodologies in a large series of 102 adult patients with symptomatic intrahepatic cholelithiasis/cholestasis. Here, we describe for the first time $A B C B 4$ partial or complete deletions in patients with LPAC and CIC.

\section{SUBJECTS AND METHODS}

\section{Patients}

A panel of 102 clinically diagnosed index cases was phenotypically selected, by routine genetic diagnosis. All patients were examined by reference hepatologists or gastroenterologists. The full clinical and radiological available information were recorded. The written informed consent from each patient included in the study was obtained. In total, 59 unrelated adult women with ICP and/or CIC and 43 unrelated adult patients with a clinical presentation compatible with LPAC syndrome were included in this present study. For the latter group, we have considered patients presenting with intrahepatic cholelithiasis despite cholecystectomy before 40 years associated with at least one of the following criteria: presence of complications (cholecystitis, cholangitis, or acute pancreatitis), intrahepatic hyperechoic foci with or without sludge or microlithiasis, response to UDCA therapy, and family history.

\section{$A B C B 4$ mutation screening}

Total DNA was extracted from whole-blood leukocytes using the Nucleon BACC2 genomic DNA extraction kit (GE Health Care Europe, Amersham, UK). Genomic DNA was amplified with primers specific for the $27 A B C B 4$ (NM_018849.2)-coding exons and their intron boundaries. The primer sequences and PCR conditions are available upon request. Mutation was identified using bidirectional DNA sequencing of purified PCR products. Sequences were aligned with Seqscape v5.1 analysis software (Applied Biosystems, Foster City, CA, USA). The ABCB4 molecular analysis was performed in the Biochemistry and Molecular Genetics Laboratory, Beaujon Hospital, Clichy, France.

\section{ABCB4 multiplex ligation-dependent probe amplification (MLPA) analysis}

The ABCB4 deletion screening was performed by MLPA analysis using the SALSA MLPA kit P109 ABCB4, as recommended in the manufacturer's protocol (MRC-Holland, Amsterdam, The Netherlands). This SALSA MLPA kit is designed to detect deletions/duplications of one or more exons of the $A B C B 4$ gene. It contains 22 probes covering $A B C B 4$-coding exons, three probes for $A B C B 4$ promoter, and three probes for the centromeric adjacent $A B C B 1$ gene. For reference, several probes for other human genes located on different chromosomes are also included. Briefly, four control samples and patient samples (each containing $100 \mathrm{ng}$ of genomic DNA) were used for overnight hybridization with the probe mixes. After ligation and amplification performed with FAM-labeled primers, PCR products were analyzed on an ABI Prism 3130 automatic DNA sequencer (Applied Biosystems). Peak areas for each separated fragment were measured by using Genemapper software v4.0 (Applied Biosystems). Normalized ratios of $<0.6$ and $>1.3$ were considered as deletions and duplications, respectively. Ratio profiles between 0.6 and 0.85 were considered as doubtful. DNA samples with $A B C B 4$ copy number variation were further analyzed by real-time PCR-based gene dosage for confirmation.

\section{Real-time PCR-based gene dosage}

We quantified $A B C B 4$ exons 10 and 11 by determining the threshold cycle $(\mathrm{Ct})$ number at which the increase in the signal associated with exponential growth of PCR products begins. We also quantified the $A L B$ gene (encoding albumin) as an endogenous DNA control, and each sample was normalized on the basis of its $A L B$ content, as previously described. ${ }^{38} A L B$ was selected as an endogenous control, because it maps to chromosome 4q11-q13, while $A B C B 4$ is at chromosome 7q21.1. The relative copy number of the $A B C B 4$ exon targets was also normalized to a calibrator, consisting of genomic DNA from a normal subject. Final results, expressed as $\mathrm{N}$-fold differences in the $A B C B 4$ exonic targets copy number relative to the $A L B$ gene and the calibrator were

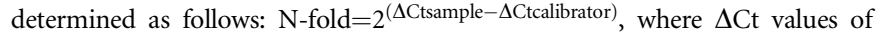
the sample and calibrator are determined by subtracting the average $\mathrm{Ct}$ value of the $A B C B 4$ exon target from the average $\mathrm{Ct}$ value of the $A L B$ gene. An $\mathrm{N}$-fold value of $<0.6$ was considered deleted. All PCRs were performed on a LightCycler 480 with the LightCycler 480 SYBR Green I Master kit (Roche Applied Science, Basel, Switzerland). PCR conditions and primer sequences are available on request. Experiments were done with triplicates for each data point.

\section{Microarray characterization of $A B C B 4$ complete deletions}

The practical aspects of array-comparative genomic hybridization (array-CGH) are described in detail elsewhere. ${ }^{39}$ Briefly, array-CGH labeling and hybridization were performed on Agilent whole human genome $400 \mathrm{~K}$ microarrays as recommended in the Agilent manual (Protocol v6.3, October 2010, Agilent Technologies, Palo Alto, CA, USA). Patients' genomic DNA and six pooled normal control DNAs (reference) were labeled with Cy5-dUTP and Cy3-dUTP, respectively. Arrays were scanned with an Agilent DNA Microarray Scanner (G2565BA). Log2 ratios were determined with Agilent Feature Extraction software (v 9.1.3.1). Results were visualized and analyzed with Agilent's Genomic Workbench 5.0 software. DNA sequence information was referred to the public UCSC (University of California Santa Cruz) database (Human Genome Browser, February 2009 assembly: hg 19, National Center for Biotechnology Information (NCBI) Build 37). 


\section{Fine characterization of $A B C B 4$ intragenic deletion breakpoints}

Long-range PCR was performed with the Expand $20 \mathrm{~kb}$ Plus PCR kit as recommended by the manufacturer (Roche Applied Science). The primer pairs and PCR conditions used to characterize the gross deletions are available upon request. The PCR products were sequenced with the ABI BigDye terminator sequencing kit (Applied Biosystems) on an ABI Prism 3130 automatic DNA sequencer (Applied Biosystems).

\section{RESULTS}

In all, 102 index cases with LPAC and ICP/CIC were screened on the basis of their phenotype for mutations in the $A B C B 4$ gene. A total of $32 A B C B 4$ heterozygous loss of function point or short insertion/ deletion mutations (including non-sense, frameshift, splice, or previously reported/predicted to be deleterious missense mutations) were identified among the 102 tested index cases: in 37\% (16/43) of LPAC patients and in $27 \%(16 / 59)$ of ICP/CIC patients.

The 70 adult patients with no $A B C B 4$ mutation found (27 with LPAC and 43 with ICP/CIC), were consequently screened for large rearrangements using MLPA. Four heterozygous deletions were identified with this approach in $7 \%(3 / 43)$ of the LPAC patients (BOC propositus of family 1 , BEA propositus of family 2 , and TIL) and in $\sim 2 \%(1 / 59)$ of the ICP/CIC patients (ROC) (Figure 1). The four deletions were confirmed using real-time PCR-based gene dosage. Clinical features of the individuals with $A B C B 4$ gene deletions (family 1: patients BOC, ARE, and BOJ; family 2: patient BEA; patient ROC; patient TIL) are summarized in Table 1. Figure 2 shows families 1 and 2 pedigrees including 12 and 5 patients with LPAC syndrome, respectively.

The four $A B C B 4$ gross deletions were then accurately characterized. They included two whole-gene deletions (TIL and ROC), and two partial intragenic deletions (BEA: exon 10 deletion and BOC: exons 11-19 deletion). Figure 3 shows characteristic array-CGH profiles for the two complete $A B C B 4$ deletions, as displayed by the workbench software. Deletion of patient ROC is flanked by last non-deleted centromeric probe at position chr7:86068686-86068745 (numbered as in build 37/hg19 assembly of the NCBI), and telomeric probe at chr7:91 $075851-$ 91075910 . Deletion of patient TIL is flanked by last non-deleted centromeric probe at chr7:86 907515-86907574 and telomeric probe at chr7:87 246 524-87246583. Patient's ROC and TIL deletions were consequently estimated to be $\sim 5 \mathrm{Mb}$ and $\sim 339 \mathrm{~kb}$ long, respectively, including 20 and 3 genes in addition to $A B C B 4$ (Table 1; Supplementary Table 1). The 400K array-CGH data on these two patients have been deposited in NCBI's Gene Expression Omnibus (GEO) and are accessible through GEO accession number GSE28676 (http://www.ncbi.nlm. nih.gov/geo/query/acc.cgi?acc=GSE28676).

The breakpoints of the two intragenic deletions (patients BEA and BOC) were precisely determined by sequencing the junction longrange PCR products. Only DNA sample from BEA yielded a 3055-bp fragment with primers JONCTIONBEA-U (5'-TGTGACTCGGAC TATGGATTGTT- ${ }^{\prime}$ ) and JONCTIONBEA-L (5'-CCAAAACTGGATT CACACGCA- $\left.3^{\prime}\right)$. Subsequent sequencing revealed that this deletion is in fact a complex rearrangement (Figure 4). A healthy control sample and BEA wild-type allele yielded a 4053-bp non-deleted fragment using the same primers. Only DNA sample from BOC (family 1 propositus) yielded a 2680 -bp fragment with primers JONCTIONBOCU (5'-AAAATAGACCCACTCAGGCAA-3') and JONCTIONBOC-L (5'-ATGCTACATGCTTATCTAAAACCAT- $\left.3^{\prime}\right)$. A healthy control sample yielded no PCR product, the wild-type allele being too large $(\sim 27 \mathrm{~kb})$ to be amplified. Sequencing the PCR product revealed a simple deletion of $24720 \mathrm{bp}$ (Supplementary Figure 1). This deletion was also detected in three other affected individuals with available DNA samples (Figure 2: individuals II:4, II:7, and II:10 of family 1).
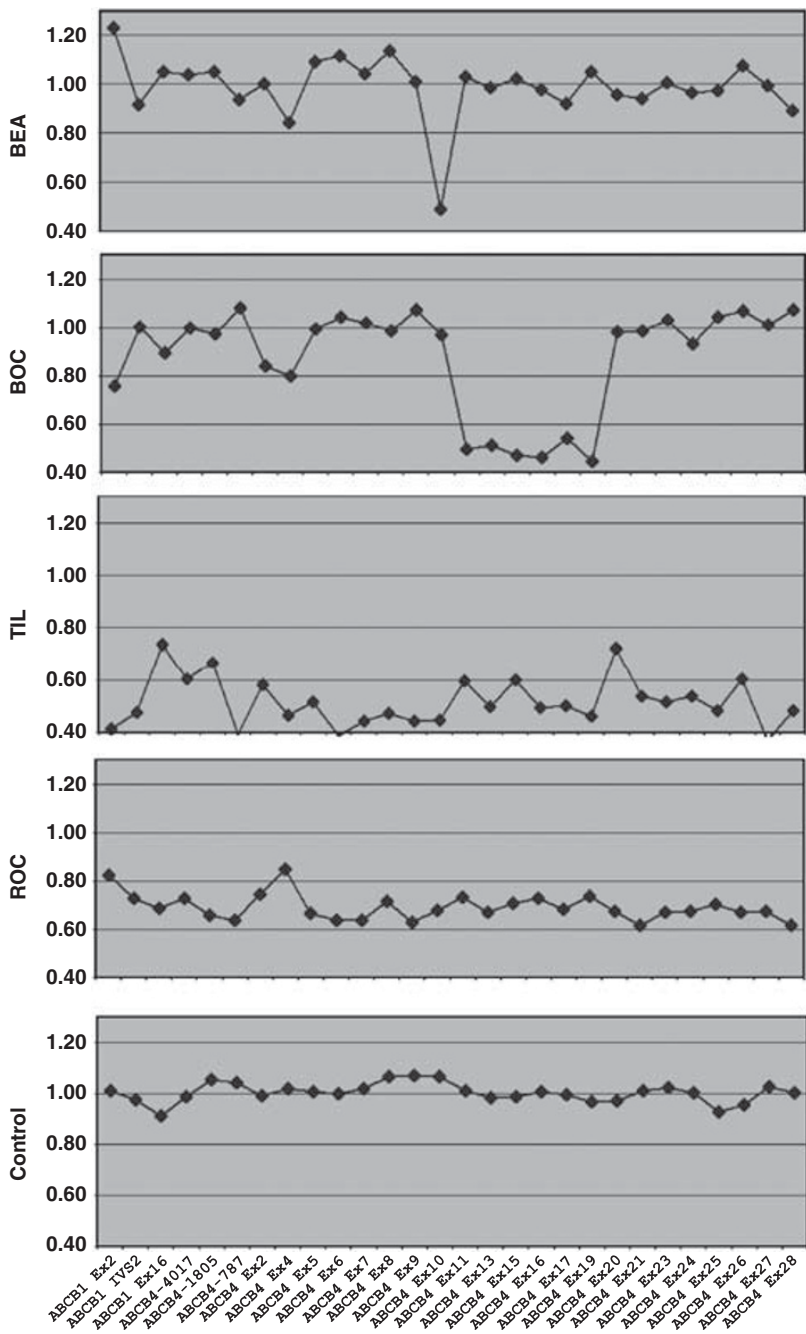

Figure 1 ABCB4 SALSA MLPA kit P109 peak areas normalized ratio profiles of patients BEA, BOC, TIL, ROC, and one normal control DNA. Two partial intragenic deletions (patients BEA and $\mathrm{BOC}$ ) and two whole-gene deletions (patients TIL and ROC) were identified. The mean of normalized ratios in patients without deletion was 1.02 .

\section{DISCUSSION}

In this study, we showed for the first time that a significant subset of patients with symptomatic cholestasis/cholelithiasis has underlying $A B C B 4$ deletions. Partial or complete heterozygous $A B C B 4$ deletions were found in $7 \%$ of the patients with LPAC and in $\sim 2 \%$ of the patients with CIC. A large family of 12 affected patients with severe LPAC and cholecystectomy (family 1) was notably reported (Table 1; Figure 2).

Recent gene dosage methodologies have allowed identification of these $A B C B 4$ deletions. Our observations urge to systematically test the patients with LPAC for the presence of $A B C B 4$ deletions. MLPA, a sensitive, rapid, and cost-effective approach seems particularly adapted to routine diagnosis in molecular genetics. We developed a molecular algorithm tailored to $A B C B 4$ routine analysis that includes $A B C B 4$ gene dosage by MLPA, in case of $A B C B 4$ negative sequencing in patients with suggestive phenotype. MLPA allows a fast and inexpensive first-line screening for both the partial and complete $A B C B 4$ deletions, complementary to a high-resolution technique such as array-CGH that can be used to characterize larger deletions. 
Table 1 Clinical and molecular characteristics of the patients carrying gross genomic deletions

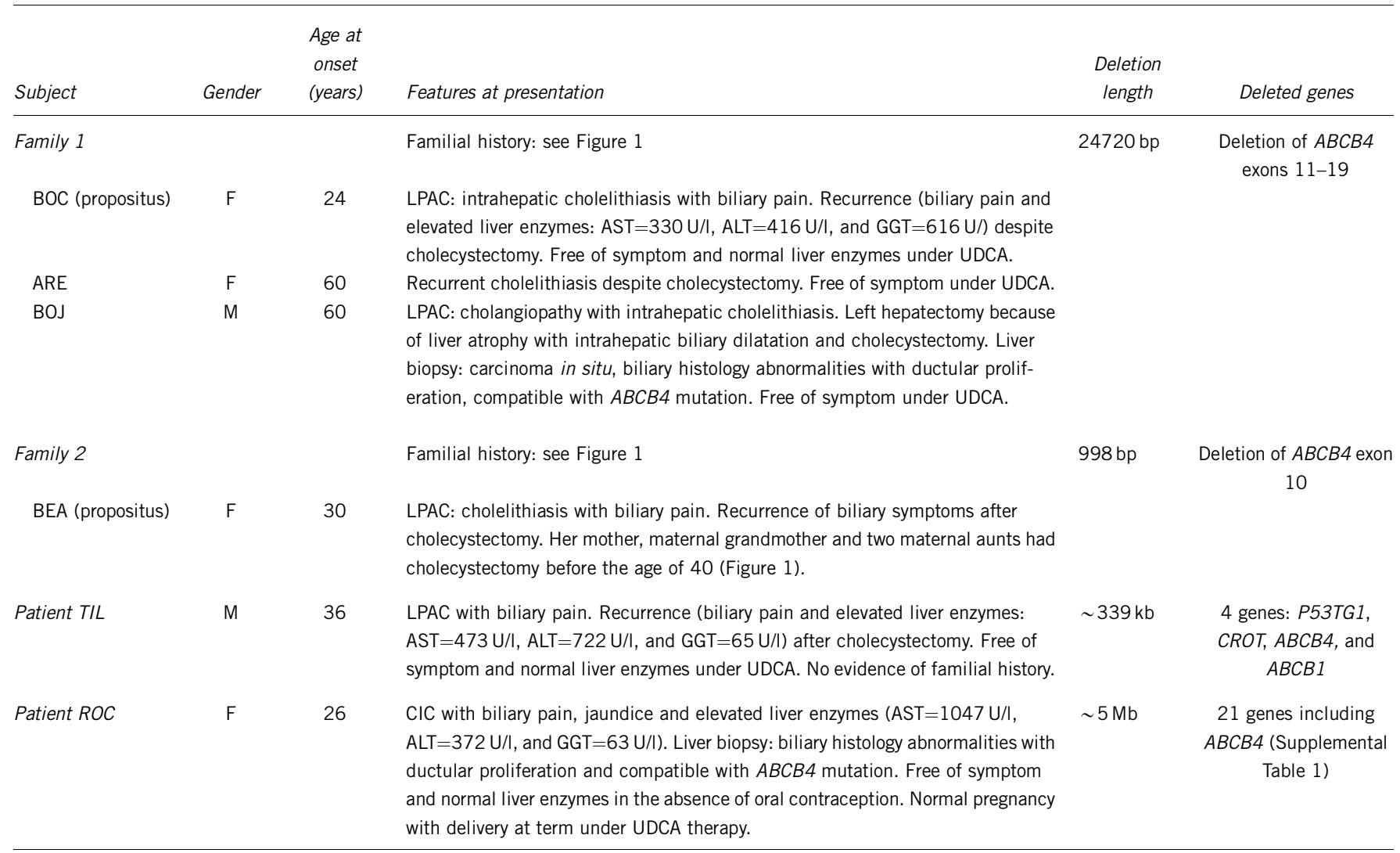

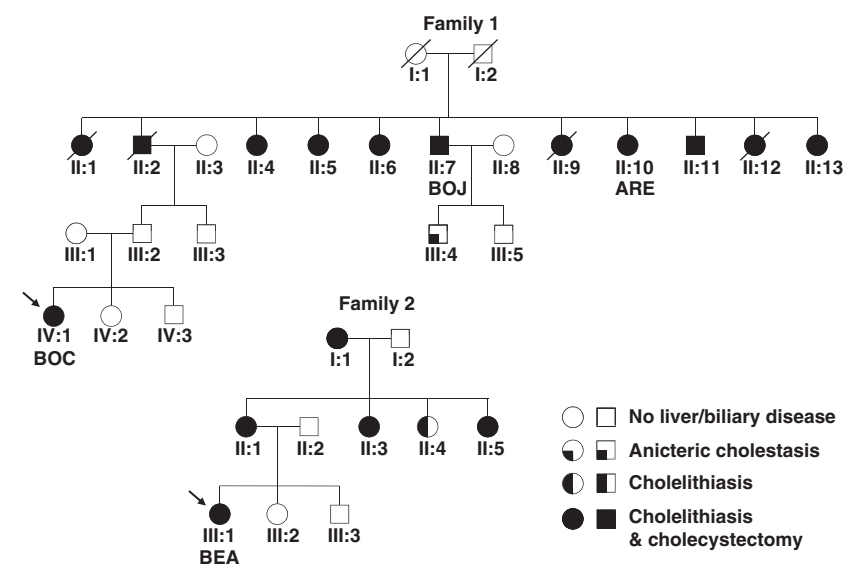

Figure 2 Pedigrees of families 1 and 2. Squares and circles indicate males and females, respectively. Clear symbols indicate unaffected individuals. Arrows indicate propositus. Individuals II:4, II:7, II:10, and IV:1 in family 1 and individual III: 1 in family 2 were molecularly tested. Individual II:2 in family 1 was diagnosed with cholangiocarcinoma at 71 years of age.

Real-time PCR-based gene dosage is useful for deletion's confirmation, particularly for the samples with ratio profiles considered as doubtful, that is, between 0.6 and 0.85 (patient ROC; Figure 1).

The two intragenic deletion breakpoints were cloned at the nucleotide level and no recurrent breakpoints were found. That no significant sequence similarity was found between the centromeric and telomeric breakpoints of both the deletions, effectively excluded homologous recombination as the underlying mutational mechanisms in both the cases. However, the presence of microhomology in each of the aberrant junctions is consistent with both the microhomologydependent replication-based recombination (MMRDR) and nonhomologous end-joining (NHEJ) mechanisms. ${ }^{40}$ Thus, the complex rearrangement detected in patient BEA can be perfectly explained by either serial replication slippage (SRS, a subpathway of MMRDR) or NHEJ repair of simultaneously generated double-strand breaks (Figure 4), while the simple $24 \mathrm{~kb}$ deletion (Supplementary Figure 1) is consistent with a single step of replication slippage (a subpathway of MMRDR), microhomology-mediated break-induced replication (MMBIR, a subpathway of MMRDR) or NHEJ. ${ }^{40}$

In this study, adult patients with LPAC were screened for the $A B C B 4$ deletions. We assume that $A B C B 4$ deletions may also be found in children with PFIC3. In some patients with PFIC3, only one $A B C B 4$ mutation or no mutation has been previously reported while no ABCB4 protein was detected by immunohistochemistry analysis. ${ }^{19}$ Some of these genotype-phenotype discrepancies could be explained by the presence of $A B C B 4$ deletions in the PFIC3 patients. However, in PFIC3 patient with no mutation found, a heterozygous $A B C B 4$ deletion would not be sufficient to explain the phenotype because PFIC3 is an autosomal recessive disease. Our observations urge to reassess the $A B C B 4$ molecular analysis in these patients using molecular tools allowing detection of rearrangements. PFIC3 patients who do respond to the UDCA therapy generally have a partial $A B C B 4$ defect (missense mutations) and the residual phospholipid concentration in the bile combined with UDCA replacement, may be sufficient 

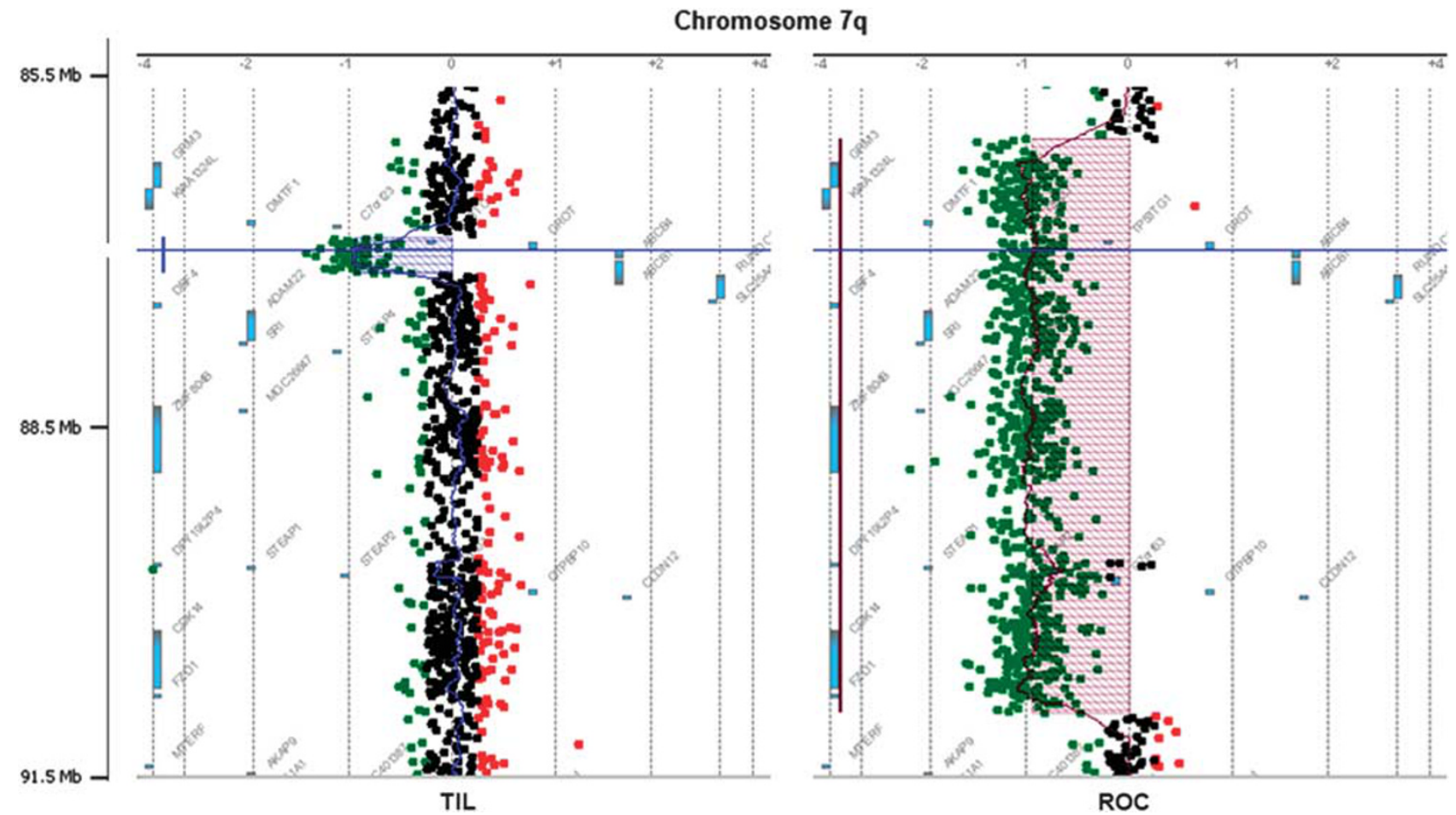

Figure 3 Array-CGH profiles of the two partially characterized $A B C B 4$ complete deletions: patients TIL and ROC. Zoom on $A B C B 4$ region at chromosome $7 \mathrm{q}$ easily identified the deletions. Horizontal blue line shows $A B C B 4$ position. Human Genome Browser, February 2009 assembly: hg 19 , NCBI Build 37.

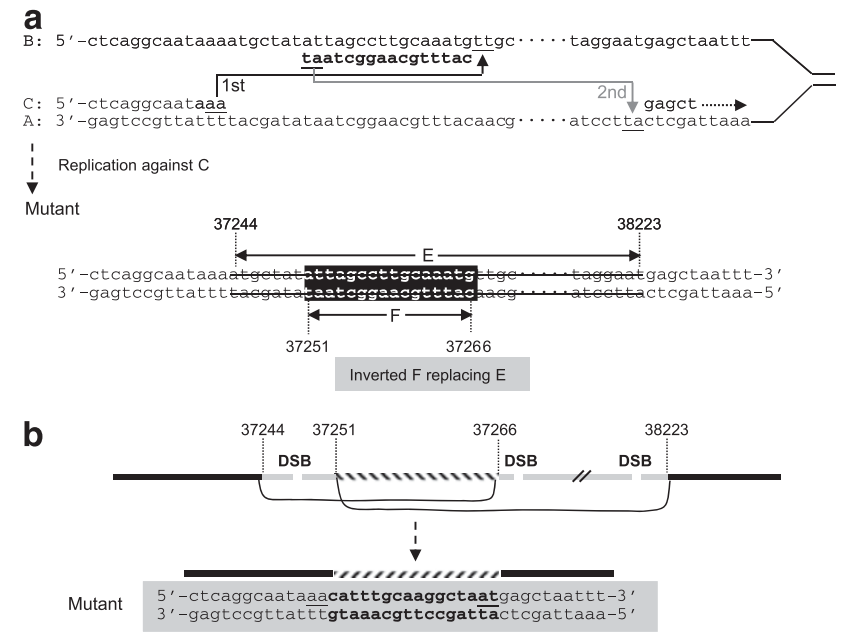

Figure 4 Illustration of two possible mechanisms underlying the complex rearrangement detected in patient BEA. (a) In the SRS model, ${ }^{42,43}$ the newly synthesized leading strand $(C)$ misaligned with the lagging template strand $(B)$ through inverted short repeats (the first step of slippage); having synthesized a short DNA sequence tract, the $C$ strand dissociated from $B$ and re-annealed to its original template strand (A) via short repeats (the second step of slippage); continued DNA synthesis (right-handed dotted arrow). Then replication against the synthesized strand $\mathrm{C}$ resulted in the observed complex rearrangement. (b) In the non-homologous end-joining (NHEJ) model, at least two double-strand breaks (DSB) occurred within the sequences deleted. One short internal sequence was re-captured in an inverted orientation during the NHEJ repair. Numbers indicating nucleotide positions are in accordance with GenBank accession EF034088.1. Short repeats are underlined.

to reduce bile salt toxicity below a critical threshold. ${ }^{19}$ ABCB4 genotyping (including deletion's detection) should help to select those PFIC3 patients who could benefit from the UDCA therapy.
In clinical practice, the establishment of a comprehensive $A B C B 4$ alteration-screening algorithm will permit the use of $A B C B 4$ genotyping, to confirm the diagnosis of LPAC syndrome in young adults who present with a symptomatic cholelithiasis and allow familial testing. One argument in support of molecular testing for $A B C B 4$ deletion is benefit of the UDCA therapy of both the symptomatic and asymptomatic cholelithiasis in patients with $A B C B 4$ deficiency. ${ }^{18}$ Depending on the results, long-term curative or prophylactic UDCA therapy could be initiated early to prevent the occurrence or recurrence of syndromes and their potential severe complications. Patient ROC who suffered from CIC may also be susceptible to the development of ICP that carries a risk of premature delivery and sudden fetal death. Identification of the $A B C B 4$ deletions may also benefit patients with CIC and ICP, as UDCA is recommended to reduce pruritus, and probably prematurity without adverse side effects. ${ }^{41}$ Management of ICP also includes close monitoring and early delivery for the fetus. All the patients with $A B C B 4$ defect in this study have benefited from the UDCA treatment. These observations are remarkable examples of the inter-relationship between molecular biology and clinical medicine.

The extreme variability and the wide spectrum of $A B C B 4$ alteration-related phenotypes make genotype-phenotype correlations difficult, although they are of crucial importance for the patients and their families. Further comparative studies of patients with well-characterized genotypes (including deletions) and phenotypes will help determine whether $A B C B 4$ mutation types influence clinical outcomes. Three of the four patients with deletions (BEA, BOC, and TIL) developed LPAC that recurred despite cholecystectomy. Patient ROC presented a very large de novo $\sim 5 \mathrm{Mb}$ deletion encompassing the $A B C B 4$ locus and 20 additional genes including $A B C B 1$ gene (Supplementary Table 1). Surprisingly, this patient presented a less severe phenotype only consisting in CIC, while being haploinsufficient for 21 genes. Unlike the $A B C B 4$ gene, none of these 20 genes is known 
to be associated with an inherited human disease according to the OMIM database (http://www.ncbi.nlm.nih.gov/omim). Variable expressions of the liver diseases caused by $A B C B 4$ mutations have previously been reported. Comorbidity factors, environmental influences, or unknown genetic modifiers may modulate these phenotypes. ${ }^{13,19}$ Our observations reinforce the potential existence of these genetic modifiers.

Gene dosage technologies have allowed the identification of $A B C B 4$ deletions in a significant subset (7\%) of patient with LPAC syndrome. An early diagnosis of this biliary disease would be beneficial because of the potential preventive effect of UDCA on the biliary complications. These data must now be taken into account in patient diagnosis and follow-up.

\section{CONFLICT OF INTEREST}

The authors declare no conflict of interest.

\section{ACKNOWLEDGEMENTS}

We thank the patients for their participation. We thank all the clinicians from France, who provided the samples for this study.

1 Oude Elferink RP, Paulusma CC, Groen AK: Hepatocanalicular transport defects: pathophysiologic mechanisms of rare diseases. Gastroenterology 2006; 130: 908-925.

2 Lincke CR, Smit JJ, van der Velde-Koerts T, Borst P: Structure of the human MDR3 gene and physical mapping of the human MDR locus. J Biol Chem 1991; 266: 5303-5310.

3 Oude Elferink RP, Paulusma CC: Function and pathophysiological importance of ABCB4 (MDR3 P-glycoprotein). Eur J Physiol 2007; 453: 601-610.

4 Lucena JF, Herrero JI, Quiroga J et al: A multidrug resistance 3 gene mutation causing cholelithiasis, cholestasis of pregnancy, and adulthood biliary cirrhosis. Gastroenterology 2003; 124: 1037-1042.

5 Kano M, Shoda J, Sumazaki R, Oda K, Nimura Y, Tanaka N: Mutations identified in the human multidrug resistance P-glycoprotein 3 (ABCB4) gene in patients with primary hepatolithiasis. Hepatol Res 2004; 29: 160-166.

6 Jung C, Driancourt C, Baussan C et al: Prenatal molecular diagnosis of inherited cholestatic diseases. J Pediatr Gastroenterol Nutr 2007; 44: 453-458.

7 Jacquemin E, de Vree JM, Cresteil D et al: The wide spectrum of multidrug resistance 3 deficiency: from neonatal cholestasis to cirrhosis of adulthood. Gastroenterology 2001; 120: $1448-1458$.

8 Rosmorduc O, Hermelin B, Boelle PY, Poupon RE, Poupon R, Chazouillères O: ABCB4 gene mutations and primary sclerosing cholangitis. Gastroenterology 2004; 126 : 1220-1222.

9 Ziol M, Barbu V, Rosmorduc 0 et al: ABCB4 heterozygous gene mutations associated with fibrosing cholestatic liver disease in adults. Gastroenterology 2008; 135: 131-141.

10 Denk GU, Bikker H, Lekanne Dit Deprez RH et al: ABCB4 deficiency: a family saga of early onset cholelithiasis, sclerosing cholangitis and cirrhosis and a novel mutation in the ABCB4 gene. Hepatol Res 2010; 40: 937-941.

11 Poupon R, Arrive L, Rosmorduc O: The cholangiographic features of severe forms of ABCB4/MDR3 deficiency-associated cholangiopathy in adults. Gastroenterol Clin Biol 2010; 34: 380-387

12 De Vree JM, Jacquemin E, Sturm E et al: Mutations in the MDR3 gene cause progressive familial intrahepatic cholestasis. Proc Natl Acad Sci USA 1998; 95: 282-287.

13 Colombo C, Vajro P, Degiorgio D et al: Clinical features and genotype-phenotype correlations in children with progressive familial intrahepatic cholestasis type 3 related to ABCB4 mutations. J Pediatr Gastroenterol Nutr 2011; 52: 73-83.

14 Degiorgio D, Colombo C, Seia M et al: Molecular characterization and structural implications of 25 new ABCB4 mutations in progressive familial intrahepatic cholestasis type 3 (PFIC3). Eur J Hum Genet 2007; 15: 1230-1238.

15 Deleuze JF, Jacquemin E, Dubuisson $C$ et al: Defect of multidrug-resistance 3 gene expression in a subtype of progressive familial intrahepatic cholestasis. Hepatology 1996; 23: 904-908.

16 Maggiore G, Bernard O, Hadchouel M, Lemonnier A, Alagille D: Diagnostic value of serum gamma-glutamyl transpeptidase activity in liver diseases in children. J Pediatr Gastroenterol Nutr 1991; 12: 21-26.
17 Rosmorduc O, Hermelin B, Poupon R: MDR3 gene defect in adults with symptomatic intrahepatic and gallbladder cholesterol cholelithiasis. Gastroenterology 2001; 120: 1459-1467.

18 Rosmorduc O, Hermelin B, Boelle PY, Parc R, Taboury J, Poupon R: ABCB4 gene mutation-associated cholelithiasis in adults. Gastroenterology 2003; 125: 452-459.

19 Davit-Spraul A, Gonzales E, Baussan C, Jacquemin E: The spectrum of liver diseases related to $A B C B 4$ gene mutations: pathophysiology and clinical aspects. Semin Liver Dis 2010; 30: 134-146.

20 Jacquemin E, Cresteil D, Manouvrier S, Boute O, Hadchouel M: Heterozygous nonsense mutation of the MDR3 gene in familial intrahepatic cholestasis of pregnancy. Lancet 1999; 353: 210-211.

21 Dixon PH, Weerasekera N, Linton KJ et al: Heterozygous MDR3 missense mutation associated with intrahepatic cholestasis of pregnancy: evidence for a defect in protein trafficking. Hum Mol Genet 2000; 9: 1209-1217.

22 Gendrot C, Bacq Y, Brechot MC, Lansac J, Andres C: A second heterozygous MDR3 nonsense mutation associated with intrahepatic cholestasis of pregnancy. J Med Genet 2003; 40: e32.

23 Müllenbach $\mathrm{R}$, Linton $\mathrm{KJ}$, Wiltshire $\mathrm{S}$ et al: $\mathrm{ABCB} 4$ gene sequence variation in women with intrahepatic cholestasis of pregnancy. J Med Genet 2003; 40: e70.

24 Pauli-Magnus $\mathrm{C}$, Kerb R, Fattinger $\mathrm{K}$ et al: BSEP and MDR3 haplotype structure in healthy Caucasians, primary biliary cirrhosis and primary sclerosing cholangitis. Hepatology 2004; 39: 779-791.

25 Keitel V, Vogt C, Häussinger D, Kubitz R: Combined mutations of canalicular transporter proteins cause severe intrahepatic cholestasis of pregnancy. Gastroenterology 2006; 131: 624-629.

26 Wasmuth HE, Glantz A, Keppeler $\mathrm{H}$ et al: Intrahepatic cholestasis of pregnancy: the severe form is associated with common variants of the hepatobiliary phospholipid transporter ABCB4 gene. Gut 2007; 56: 265-270.

27 Hay JE: Liver disease in pregnancy. Hepatology 2008; 47: 1067-1076.

28 Schneider G, Paus TC, Kullak-Ublick GA et al: Linkage between a new splicing site mutation in the MDR3 alias ABCB4 gene and intrahepatic cholestasis of pregnancy. Hepatology 2007; 45: 150-158.

29 Floreani A, Carderi I, Paternoster D et al: Intrahepatic cholestasis of pregnancy; three novel MDR3 gene mutations. Aliment Pharmacol Ther 2006; 23: 1649-1653.

30 Floreani A, Carderi I, Paternoster D et al: Hepatobiliary phospholipid transporter ABCB4, MDR3 gene variants in a large cohort of Italian women with intrahepatic cholestasis of pregnancy. Dig Liver Dis 2008; 40: 366-370.

31 Bacq $\mathrm{Y}$, Gendrot $\mathrm{C}$, Perrotin $\mathrm{F}$ et al: ABCB4 gene mutations and single-nucleotide polymorphisms in women with intrahepatic cholestasis of pregnancy. J Med Genet 2009; 46: 711-715.

32 Mazzella G, Rizzo N, Azzaroli F et al: Ursodeoxycholic acid administration in patients with cholestasis of pregnancy: effects on primary bile acids in babies and mothers. Hepatology 2001; 33: 504-508.

33 Paus TC, Schneider G, Van De Vondel P V, Sauerbruch T, Reichel C: Diagnosis and therapy of intrahepatic cholestasis of pregnancy. Z Gastroenterol 2004; 42: 623-628.

34 Pauli-Magnus C, Meier PJ: Hepatobiliary transporters and drug-induced cholestasis. Hepatology 2006; 44: 778-787.

35 Ganne-Carrié N, Baussan C, Grando V, Gaudelus J, Cresteil D, Jacquemin E: Progressive familial intrahepatic cholestasis type 3 revealed by oral contraceptive pills. J Hepatol 2003; 38: 693-694.

36 Lang C, Meier Y, Stieger B et al: Mutations and polymorphisms in the bile salt export pump and the multidrug resistance protein 3 associated with drug-induced liver injury. Pharmacogenet Genomics 2007; 17: 47-60.

37 Poupon R, Barbu V, Chamouard P, Wendum D, Rosmorduc O, Housset C: Combined features of low phospholipid-associated cholelithiasis and progressive familial intrahepatic cholestasis 3. Liver Int 2010; 30: 327-331.

38 Pasmant E, de Saint-Trivier A, Laurendeau I, Dieux-Coeslier A, Parfait B, Vidaud M et al: Characterization of a 7.6-Mb germline deletion encompassing the NF1 locus and about a hundred genes in an NF1 contiguous gene syndrome patient. Eur J Hum Genet 2008; 16: 1459-1466

39 Pasmant E, Sabbagh A, Masliah-Planchon J et al: Detection and characterization of NF1 microdeletions by custom high resolution array CGH. J Mol Diagn 2009; 11 : 524-529.

40 Chen JM, Cooper DN, Férec C, Kehrer-Sawatzki H, Patrinos GP: Genomic rearrangements in inherited disease and cancer. Semin Cancer Biol 2010; 20: 222-233.

41 Hardikar W, Kansal S, Oude Elferink RP, Angus P: Intrahepatic cholestasis of pregnancy: when should you look further? World J Gastroenterol 2009; 15: 1126-1129.

42 Chen JM, Chuzhanova N, Stenson PD, Férec C, Cooper DN: Complex gene rearrangements caused by serial replication slippage. Hum Mutat 2005; 26: 125-134.

43 Chen JM, Chuzhanova N, Stenson PD, Férec C, Cooper DN: Intrachromosomal serial replication slippage in trans gives rise to diverse genomic rearrangements involving inversions. Hum Mutat 2005; 26: 362-373.

Supplementary Information accompanies the paper on European Journal of Human Genetics website (http://www.nature.com/ejhg) 